\title{
A Pediatric COVID19 Case with Suspected Acute Abdomen, Hyperferritinemic Sepsis and Developing MIS-C and Pancreatitis
}

\author{
Nagehan Aslan ${ }^{1}$ (D) $\cdot$ Dincer Yildizdas $^{2}$ (D) $\cdot$ Muhammed Selcuk Sinanoglu $^{1}$ (D)
}

Received: 16 September 2020 / Accepted: 13 October 2020 / Published online: 22 October 2020

(C) Dr. K C Chaudhuri Foundation 2020

To the Editor: Multisystem inflammatory syndrome in children (MIS-C), that develops in children due to COVID-19 is a rare but serious condition associated with COVID-19 reported in children [1].

A healthy 12-y-old female patient had been admitted with abdominal pain, vomiting and fever and hospitalized with a prediagnosis of acute appendicitis. Surgical intervention decision was abandoned in the patient whose diarrhea started. SARS CoV2 RT-PCR test was positive. Abdominal CT revealed multiple lymphadenopathies with an edematous appearance compatible with typhlitis in terminal ileum. Broad-spectrum antibiotics and antiviral therapies were administered. COVID-19associated MIS-C was considered in the patient. Because the patient's fever was above $38^{\circ} \mathrm{C}$ and was resistant to antipyretics, there was no decrease in infectious parameters, his lymphopenia did not improve; albumin level did not increase despite albumin replacement, and respiratory and gastrointestinal symptoms continued. IVIG $2 \mathrm{~g} / \mathrm{kg}$ was administered as a $12-\mathrm{h}$ infusion. The patient's fever decreased dramatically after IVIG infectious parameters regressed. Pancreatitis developed on the 4th day. The patient was discharged on the 13th day.

In a pediatric series, all 8 cases presented with atypical appendicitis [2]. Radiological imaging revealed lymphadenopathy in the abdomen and terminal ileitis in all children. Three of them required inotropic support in PICU. Four patients suspected of MIS-C were given IVIG. In India, an 11-y-old patient presented with fever, abdominal pain and skin rash [3]. Radiological imaging revealed inflammation in the terminal ileum and cecum.

Nagehan Aslan

nagehan_aslan@hotmail.com

1 Department of Pediatric Intensive Care, Malatya Education and Research Hospital, Malatya, Turkey

2 Department of Pediatric Intensive Care, Cukurova University Medical Faculty, Adana, Turkey
SARS CoV2 RT-PCR test was positive, and clinical improvement was observed with IVIG treatment.

Elevated ferritin levels were associated with increased mortality in COVID-19 patients [4]. Management of hyperferritinemic sepsis can differ from sepsis without hyperferritinemia. In addition to the usual antimicrobial approach, one should consider administration of specific antivirals, antiparasitics, antibacterials, and antifungals; as well as non-specific neutralization with IVIG for infections without specific therapies in the hyperferritinemic patient [5].

Although it is not clear whether pancreatitis in our case was related to drugs or developed as a part of the gastrointestinal presentation of COVID-19. Our case is important in terms of drawing attention that COVID-19 may present with a picture that mimics acute appendicitis in children.

\section{Compliance with Ethical Standards}

Conflict of Interest None.

\section{References}

1. Riphagen S, Gomez X, Gonzalez-Martinez C, Wilkinson N, Theocharis P. Hyperinflammatory shock in children during COVID-19 pandemic. Lancet. 2020;395:1607-8.

2. Tullie L, Ford K, Bisharat M, et al. Gastrointestinal features in children with COVID-19: an observation of varied presentation in eight children. Lancet Child Adolesc Health. 2020;4:e19-20.

3. Periyakaruppan M, Kumar S, Kandasamy S, Thiagarajan A, Sangaralingam T, Ganapathy N. COVID abdomen: SARS-CoV-2 infection presenting as 'acute abdomen' in a child. Crit Care Emerg Med. 2020. https://doi.org/10.21203/rs.3.rs-35865/v1.

4. Zhou F, Yu T, Du R, et al. Clinical course and risk factors for mortality of adult inpatients with COVID-19 in Wuhan, China: a retrospective cohort study. Lancet. 2020;395:1054-62.

5. Carcillo JA, Kernan KK, Horvat CM, Simon DW, Aneja RK. Why and how is hyperferritinemic sepsis different from sepsis without hyperferritinemia? Pediatr Crit Care Med. 2020;21:509-12.

Publisher's Note Springer Nature remains neutral with regard to jurisdictional claims in published maps and institutional affiliations. 